\title{
TRANSIENT ISCHAEMIC ATTACK IN PREGNANCY DIAGNOSED AS PRIMARY ANTIPHOSPHOLIPID ANTIBODY SYNDROME
}

\author{
Kalaivani Kannan ${ }^{1}$, Revathi Rajamurthy² \\ 1 Professor, Department of Obstetrics and Gynaecology, Government Stanley Medical College. \\ ${ }^{2}$ Postgraduate, Department of Obstetrics and Gynaecology, Government Stanley Medical College.
}

\section{ABSTRACT}

We report the case of young primiparous women with transient ischaemic attack diagnosed as primary antiphospholipid antibody syndrome. Lupus anticoagulant and anticardiolipin antibodies were elevated. There was no clinical or laboratory evid ence for other autoimmune or systemic illnesses. We are presenting the case due to the rarity of the same.

\section{KEYWORDS}

APLA, TIA, Lupus Anticoagulant, Anticardiolipin Antibodies.

HOW TO CITE THIS ARTICLE: Kannan K, Rajamurthy R. Transient ischaemic attack in pregnancy diagnosed as primary antiphospholipid antibody syndrome. J. Evolution Med. Dent. Sci. 2016;5(66):4753-4755, DOI: 10.14260/jemds/2016/1083

\section{INTRODUCTION}

Antiphospholipid antibodies are a heterogeneous group of autoantibodies directed against negatively charged phospholipids or phospholipid-binding proteins. Primary antiphospholipid syndrome occurs in patients without clinical evidence of another autoimmune disease whereas secondary antiphospholipid syndrome occurs in association with other autoimmune disease.

\section{Case Report}

25 years pregnant primiparous women with 33 weeks of gestation presented to our OPD with history of giddiness, slurred speech, and weakness of her right upper and lower limbs for 10 minutes duration. She had similar complaints one week back lasted for 15 minutes without loss of consciousness. She did not have dysphagia, diplopia, or bladder and bowel disturbances. Past medical history was unremarkable and there was no significant family history suggestive of hereditary neurological condition. On examination, she was conscious, oriented, grade 4 power of right upper and lower limbs and dysarthria present. As the patient presented as transient ischaemic attack we considered the possibility of a hypercoagulable state.

\section{Investigations}

Investigation revealed the following findings: Blood routine, urine routine, serum electrolytes, renal, liver and thyroid function, ECG were normal. ECHO was normal study. Platelet count was 2.5 lakhs. Lipid profile was: Total cholesterol 288, Triglycerides 527, HDL 59, VLDL 105. Prothrombin time 14 seconds, Activated partial thromboplastin time 28 seconds, INR 1.3.

\section{MRI Brain and MR Angiogram}

1) Early subacute infarcts involving left MCA territory and left MCA-PCA transformation zone. No haemorrhagic transformation/mass effect seen. 2) $M_{1}$ segment of left MCA narrowed in caliber with poorly-visualised $M_{2}$ and $M_{3}$ segment ? thrombosis. 3) Normal flow void is seen in the major dural venous sinuses. 4) No evidence of any intracranial haemorrhage. Doppler study of 4 neck vessels normal. Foetal Doppler study was normal.

Financial or Other, Competing Interest: None.

Submission 12-02-2016, Peer Review 01-08-2016,

Acceptance 09-08-2016, Published 18-08-2016.

Corresponding Author:

Dr. Revathi Rajamurthy,

D/o. S. Rajamurthy, 172/2 Road Street,

Sirumur Village and Post,

Arni-632314, Tiruvannamalai.

E-mail:ssr.revathi@gmail.com

DOI: $10.14260 /$ jemds/2016/1083

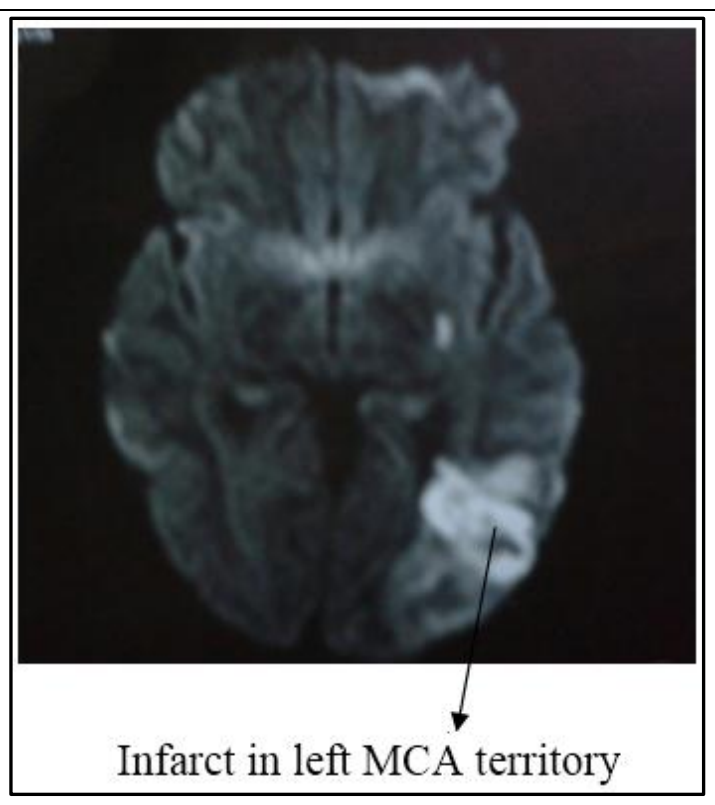

Poorly-visualised $\mathrm{M}_{2}$ and $\mathrm{M}_{3}$ segment in left MCA territory

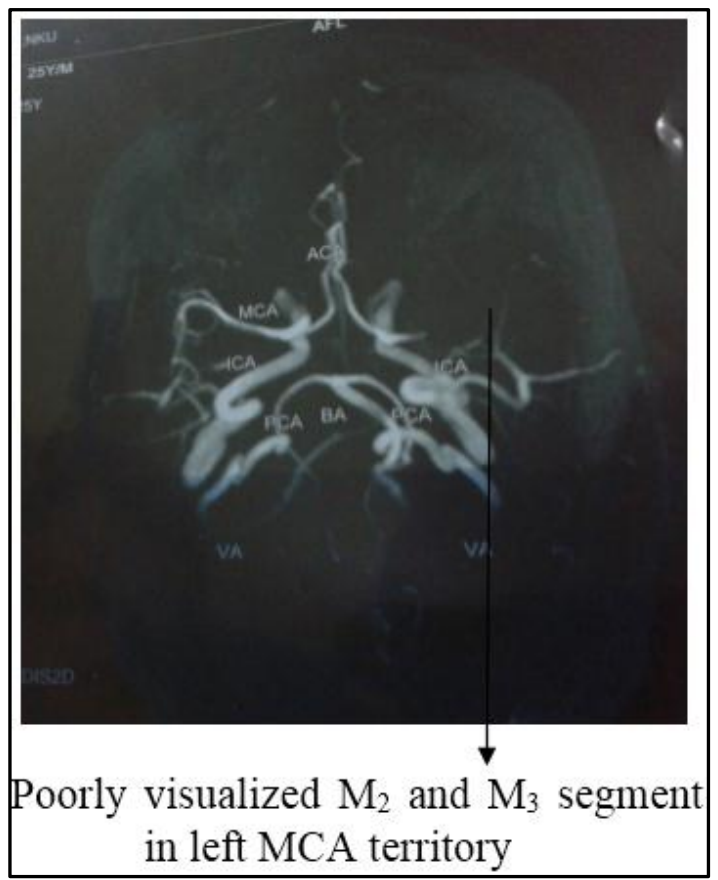

ANA, Anti ds DNA, ENA profile, and VDRL tests were negative. 
Antiphospholipid Antibody Titres were as Follows

1. Lupus anticoagulant test were done, which was prolonged by using a PTT and diluted Russell Viper Venom Time.

2. Anticardiolipin antibodies are measured by ELISA method, which was (45 MPL units $>99$ th percentile).

3. Anti-beta 2 glycoprotein 1 antibodies-Negative.

Procoagulant workup including protein S, protein C, antithrombin, urine homocysteine assays were normal. Factor $\mathrm{V}$ Leiden mutation was not done.

\section{Management and Outcome}

Based on the clinical and laboratory findings, we made a definite diagnosis of primary APLA syndrome. We started her on low molecular weight heparin ENOXAPARIN $60 \mathrm{mg}$ s.c. b.d. and T. Aspirin $300 \mathrm{mg}$ stat followed by $150 \mathrm{mg}$ once a day. Biweekly non-stress test and AFI measured. On 37 weeks 6 days, she had persistent decreased foetal movements. Heparin was stopped for $24 \mathrm{hrs}$. Then, emergency LSCS was done. She delivered an alive term boy baby of wt. $3.1 \mathrm{~kg}$ with good Apgar. 1 unit of packed red blood cell and 4 unit of FFP transfused intraoperatively. Heparin restarted after $12 \mathrm{hrs}$. Heparin was switched over to oral anticoagulant on $5^{\text {th }}$ postoperative day. INR maintained between 2-3. Postoperative period was uneventful. Oral anticoagulant continued till 6 weeks postpartum. IgM anticardiolipin antibody was repeated after 12 weeks and it came out to be positive (42 MPL units >99th percentile), Lupus anticoagulant test was negative after 12 weeks.

\section{DISCUSSION}

Antiphospholipid Antibody Syndrome (APLA, APS, Hughes syndrome) is characterised by thrombosis or foetal loss in association with presence of Lupus Anticoagulant [LA] or anticardiolipin antibodies. Important obstetric criteria include recurrent pregnancy loss, foetal death, severe preeclampsia, or placental insufficiency requiring delivery prior to 34 weeks gestation. Pregnancy complications due to APS are due to abnormal placental function. This results in narrowing of the spiral arterioles, intimal thickening, acute atherosis, and fibrinoid necrosis. Extensive necrosis, infarction, and thrombosis are seen in the placenta.

Venous thrombosis, especially deep venous thrombosis of the legs, is the most common manifestation of the antiphospholipid syndrome occurring in 29 to 55 percent of patients with the syndrome during an average followup of less than six years. ${ }^{1}$ Arterial thromboses are less common than venous thromboses and most frequently manifest with features consistent with ischaemia or infarction. ${ }^{1}$ The brain is the most common site with strokes and transient ischaemic attacks accounting for almost 50 percent of arterial occlusions. $^{2}$ Other prominent manifestations of the antiphospholipid syndrome include thrombocytopenia (in 40 to 50 percent of patients), haemolytic anaemia (in 14 to 23 percent), and Livedo reticularis (in 11 to 22 percent). ${ }^{1}$ Since pregnancy itself is a hypercoagulable state presence of aPL antibodies can precipitate any of the above-mentioned complications at any time either during pregnancy or during postpartum state. ${ }^{3}$ So, in short, suspect the presence of aPL antibodies when there is clinical symptoms such as DVT, arterial occlusive events, recurrent foetal loss, TIA, etc.
Gestational hypertension/preeclampsia occurs in about $30 \%$ of APS pregnancies. Preeclampsia is usually more severe in APS pregnancies. ${ }^{4}$ aPLs frequently cause IUGR in almost one in three pregnancies with APS. Preterm birth rates are high ranging from $30-60 \%$ in APS pregnancies. APS related pregnancy loss could be an early recurrent pregnancy loss. Usually, it is an embryonic foetal loss.

International consensus statement on preliminary criteria for the classification of antiphospholipid antibody syndrome is as follows.

\section{Revised Sapporo Criteria for the Diagnosis of Definite Antiphospholipid Syndrome}

APS is present if at least one of the clinical and one of the laboratory criteria that follow are met.

\section{Clinical}

A documented episode of arterial, venous, or small vessel thrombosis-other than superficial venous thrombosis - in any tissue or organ by objective validated criteria with no significant evidence of inflammation in the vessel wall and/or 1 or more unexplained deaths of a morphologically normal foetus (documented by ultrasound or direct examination of the foetus) at or beyond the 10th week of gestation and/or 3 or more unexplained consecutive spontaneous abortions before the 10th week of gestation with maternal anatomic or hormonal abnormalities and paternal and maternal chromosomal causes excluded or at least 1 premature birth of a morphologically normal neonate before the 34th week of gestation due to eclampsia or severe preeclampsia according to standard definitions or recognised features of placental insufficiency plus.

\section{Laboratory}

- Anticardiolipin IgG and/or IgM measured by standardised, non-cofactor dependent ELISA on 2 or more occasions, not less than 12 weeks apart; medium or high titre (i.e., $>40$ GPL or MPL, or $>$ the $99^{\text {th }}$ percentile) and/or,

- Anti- $\beta 2$ glycoprotein 1 IgG and/or IgM measured by standardised ELISA on 2 or more occasions, not less than 12 weeks apart; medium or high titre (>the 99th percentile) and/or,

- Lupus anticoagulant detected on 2 occasions not less than 12 weeks apart according to the guidelines of the International Society of Thrombosis and Haemostasis.

At present, the therapeutic prevention of obstetric accidents related to APAS saves $70 \%$ of survival births compared to $10 \%$ without treatment.[5] Several treatments were studied, in particular aspirin, heparin, prednisone, immunoglobulin, and plasmapheresis. However, most authors indicate the association of aspirin and heparin at low molecular weight. [5-12] Indeed, this association therapy allows reducing the foetal loss by $54 \%$. Actually, most studies have shown that the best therapy of APAS is offered by aspirin (60 to $100 \mathrm{mg}$ ) and heparin of LMW (An injection a day in preventive dose). Prednisone are suggested only if there is an extra-obstetrical[5] or inflammatory system disease, this without exceeding $20 \mathrm{mg} /$ day. Immunoglobulins generate a solubilisation of the immune complexes. However, a randomised study didn't show any profit of using IgG compared a placebo in patients treated by aspirin and heparin 
particularly on the rate of children survival and the prematurity.[13] Plasmapheresis wasn't studied in the treatment of APAS during the pregnancy. Besides these treatments, an obstetric care based on the close observation is essential.

\section{REFERENCES}

1. Levine JS, Branch DW, Rauch J. The antiphospholipid syndrome. N Engl J Med 2002;346(10):752-63.

2. Ameri A, Bousser M. Cerebral venous thrombosis. Neurol Clin 1992;10(1):87-111.

3. Myons BL, Mccurdy D. APLA syndrome. Medicine Update 2002:4.

4. Moodiey J, Bhoola V, Duursma J, et al. The association of antiphospholipid antibodies with severe early onset preeclampsia. S Afr Med J 1995;85:105-7.

5. Meyer O. Lupus et autres connectivites et vie hormonale. Gynaecologie Obstetrique and Fertilite 2003;31(9): 746-56.

6. Carbonne B, Lejeune V. Modalites therapeutiques d'une maladie abortive d'origine dysimmunitaire. Gynaecologie Obstetrique and Fertilite 2003;31:789-93.

7. Arvieux J, Hachulla E. Le syndrome des antiphospholipides. Annales de Cardiologie et d Angeiologie 2002;51:146-51.
8. Firkin BG, Howard MA, Radford N. Possible relationship between lupus inhibitor and recurrent abortion in young women. Lancet 1980;2(8190):366.

9. Lejeune V. Fausses couches spontanees precoces repetees: quelleprise encharge proposer en 2006? Gynaecologie Obstetrique and Fertilite 2006;34:927-93.

10. Nielsen HS, Christiansen OB. Prognostic impact of anticardiolipin antibodies in women with recurrent miscarriage negative for the lupus anticoagulant. Human Reproduction 2005;20(6):1720-8.

11. Wilson WA, Gharavi $\mathrm{AE}$, Koike $\mathrm{T}$, et al. International consensus statement on preliminary classification criteria for definite antiphospholipid syndrome. Arthritis and Rheumatism 1999;42(7):1309-11.

12. Maistre DE. Anticorps dits antiphospholipides et troubles de la reproduction: les donnees biologiques. Gynaecologie Obstetrique and Fertilite 2003;31:794-6.

13. Branch DW, Peaceman AM, Druzin M, et al. A multicenter, placebo-controlled pilot study of intravenous immune globulin treatment of antiphospholipid syndrome during pregnancy. The pregnancy loss study group. American Journal of Obstetrics and Gynaecology 2000;182(1 Pt 1):122-7. 